\title{
Miscible Blends of Stereoregular Poly(methyl methacrylate) and Poly(hydroxy ether of Bisphenol A)
}

\author{
Wen-Ping $\mathrm{HsU}^{\dagger}$ and Ching-Fen YEH \\ Department of Applied Chemistry, Chia-Nan College of Pharmacy and Science, \\ \#60 Sec. 1 Erh-Jen Road, Jen-Te Hsian, Tainan Taiwan, Republic of China 71710
}

(Received June 24, 1999)

\begin{abstract}
Isotactic, atactic, and syndiotactic poly(methyl methacrylates) (PMMAs) (designated as iPMMA, aPMMA, and sPMMA) were mixed with poly(hydroxy ether of Bisphenol A) (phenoxy) separately in 2-butanone to make three polymer blend systems. Differential scanning calorimetry (DSC) and Fourier transform infrared (FT-IR) spectroscopy were used to study the miscibility of these blends. The three polymer blends were found to be miscible because all the prepared films were transparent and there was a single glass transition temperature $\left(T_{\mathrm{g}}\right)$ for each composition of the blends. $T_{\mathrm{g}}$ values of the sPMMA/phenoxy blends correspond well to weight average predictions. Since $T_{\mathrm{g}}$ values of aPMMA and phenoxy are close, naturally their blends' $T_{\mathrm{gs}}$ are not different from weight average. However, $T_{\mathrm{g}}$ values of the iPMMA/phenoxy blends are below weight average and can be fitted well by the Gordon-Taylor equation with a $k$ value of 0.38 . The IR spectra of carbonyl absorption showed little hydrogen bonding since virtually no shift was observed. However, the spectra in the region of hydroxyl absorption indicated a certain degree of hydrogen bonding between PMMA and phenoxy. Upon mixing with PMMA, the broad hydrogen bonded hydroxyl band of the phenoxy was observed to shift to higher frequencies as a function of increasing PMMA concentration. Using the magnitude of the peak shift of the hydrogen bonded hydroxyl band as an indicator of interaction between PMMA and phenoxy, sPMMA was determined to have the highest degree of hydrogen bonding with phenoxy among the three tactic PMMAs. Based on the results of IR spectra, the intra-hydrogen bonding $(\mathrm{OH}-\mathrm{OH})$ interaction of phenoxy was found to be stronger than the interhydrogen bonding $(\mathrm{OH}-\mathrm{O}=\mathrm{C})$ interaction between PMMA and phenoxy.
\end{abstract}

KEY WORDS Miscible Blends / Stereoregular / Poly(methyl methacrylate)/ Phenoxy /

It has been known for years that the stereoregularity of polymer chains influences polymer-polymer miscibility. Due to its availability in both syndiotactic and isotactic forms, poly(methyl methacrylate) (PMMA) has been used frequently in the investigation of the effect of tacticity on miscibility. Several papers ${ }^{1-8}$ have shown that the tacticity of PMMA influences blend miscibility, when PMMA is blended with a chemically different polymer. Because of differences in the molecular weights and the preparation methods of samples, the results sometimes are not consistent. Since atactic PMMA is mainly syndiotactic, the results of atactic and syndiotactic are often similar.

Phenoxy, which is a poly(hydroxy ether of bisphenol A), is a relatively tough and ductile thermoplastic with an excellent oxygen barrier property. According to literature, phenoxy is miscible with aromatic or aliphatic polyesters such as poly(butylene terephthalate $)^{9,10}$, and poly(caprolactone), ${ }^{11}$ and with polyethers such as poly(ethylene oxide). ${ }^{12}$ The miscibility is mainly due to strong hydrogen bonding between the component polymers. That is, the hydroxyl group in phenoxy acts as a proton donor while the ester or ether group of the other polymers acts as a proton acceptor, and their combinations result in thermodynamically miscible blends.

It is known that phenoxy is immiscible with most thermoplastics. However, for PMMA/phenoxy blends, Chiou and Paul ${ }^{13}$ reported a single composition dependent glass transition temperature and lower critical solution temperature (LCST) behavior. Blends of PMMA and phenoxy have been also prepared by melt extrusion using a single screw extruder. ${ }^{14}$ The injection molded

\footnotetext{
${ }^{\dagger}$ To whom all correspondence should be addressed.
}

specimens were transparent, and differential scanning calorimetry and rheological measurements detected a single glass transition temperature $\left(T_{\mathrm{g}}\right)$. Therefore, phenoxy and PMMA were determined to be miscible based on the results of ref 13 and 14.

This research was prompted by the idea that atactic PMMA and phenoxy are miscible. How about the miscibility between isotactic or syndiotactic PMMA and phenoxy? Also to our knowledge, no one has reported a systematic study on the tacticity of PMMA on its miscibility with phenoxy. Therefore, this topic was pursued in our laboratory.

In this article, isotactic, atactic and syndiotactic PMMAs with almost the same molecular weight were blended with phenoxy in 2-butanone to cast into films. The glass transition temperatures of the polymers were measured and FT-IR spectra of the polymers were conducted to study the degree of hydrogen bonding between different tactic PMMAs and phenoxy. In this report, the miscibility of the prepared blends is investigated based on the data of glass transition temperatures and FT-IR spectra.

\section{EXPERIMENTAL}

\section{Materials}

Isotactic, atactic, and syndiotactic PMMAs (designated as i, a, and sPMMA in this study) were purchased from Polysciences, Inc., Warrington, PA. According to the supplier information, the molecular weights $\left(M_{\omega}\right)$ of iPMMA, aPMMA, and SPMMA are the same about 100000. The poly(hydroxy ether of Bisphenol A) (phenoxy) used for this study was obtained from Scientific Polymer Products, Inc., Ontario, N.Y. The $M_{w}$ value for 
Table I. Glass transition temperatures of PMMA/phenoxy blends

\begin{tabular}{cccc}
\hline & $T_{\mathrm{gsc}} /{ }^{\circ} \mathrm{C}$ & $T_{\mathrm{gq}} /{ }^{\circ} \mathrm{C}$ & $\Delta T_{\mathrm{gq}} /{ }^{\circ} \mathrm{C}$ \\
\hline (1) iPMMA/Phenoxy & & & \\
100/0 & 71.9 & 74.6 & 20 \\
$75.1 / 24.9$ & 72.6 & 77.6 & 17 \\
$50.0 / 50.0$ & 78.1 & 80.9 & 15 \\
25.0/75.0 & 87.8 & 88.0 & 14 \\
(2) aPMMA/Phenoxy & & & \\
100/0 & 103.4 & 102.7 & 12 \\
$75.5 / 24.5$ & 103.3 & 102.2 & 13 \\
49.9/50.1 & 104.0 & 101.1 & 11 \\
$25.1 / 74.9$ & 101.2 & 101.2 & 9 \\
(3) sPMMA/Phenoxy & & & \\
100/0 & 121.4 & 122.4 & 13 \\
$75.2 / 24.8$ & 119.9 & 119.3 & 16 \\
$49.8 / 50.2$ & 110.3 & 111.9 & 16 \\
$23.7 / 76.3$ & 102.0 & 103.0 & 11 \\
$0 / 100$ &.-- & 99.5 & 10 \\
\hline
\end{tabular}

phenoxy is 70000 . Phenoxy was mixed with each tactic PMMA individually to form blends in the weight ratios of about $1 / 3,1 / 1$, and $3 / 1$. The actual compositions of the polymer blends are shown in Table I.

\section{Film Preparation}

Thin films of individual polymers and their blends were made by solution casting onto glass plates. 2Butanone was used as solvent for most compositions, but for PMMA toluene was used. 2-Butanone and toluene are A.C.S. reagent purchased from Aldrich Chemical Company, Inc., Milwaukee, WI and Fisher Scientific, Fair Lawn, New Jersey, respectively. The final drying step for all the films took place in a vacuum oven for about 1 day at $92-125^{\circ} \mathrm{C}$, which was above the glass transition temperatures of the polymers. Then the films were cooled to room temperature slowly by air. The ascast films were then used for DSC studies.

\section{Differential Scanning Calorimetry (DSC) and Fourier}

\section{Transform Infrared (FT-IR) Spectroscopy}

The glass transition temperatures $\left(T_{\mathrm{g}} \mathrm{s}\right)$ of the polymer blends were determined by using a DuPont 2000 thermal analyzer. The scanning range for temperature was from 30 to $200^{\circ} \mathrm{C}$ and a heating rate of $20^{\circ} \mathrm{C} \mathrm{min}{ }^{-1}$ was used in every measurement. The experiments were performed in two consecutive scans in the ambient environment of nitrogen gas at a flowing rate of about $100 \mathrm{ml}$ $\min ^{-1}$. At the end of the first thermal scan, the samples stayed at $200^{\circ} \mathrm{C}$ for $1 \mathrm{~min}$. Then the samples were quenched to $0^{\circ} \mathrm{C}$ immediately using an ice-water bath. The samples were quenched quickly enough to prevent any crystallization as detected by DSC. There was also no trace of solvent in the films detectable by DSC. The inflection point of the specific heat jump of a thermal scan was taken as the glass transition temperature. The glass transition temperatures determined from the first and second thermal scans are designated as $T_{\mathrm{gsc}}$ and $T_{\mathrm{gq}}$, respectively. Therefore, $T_{\mathrm{gsc}}$ is the $T_{\mathrm{g}}$ of the slowly cooled (as-cast) films and $T_{\mathrm{gq}}$ is that of the quenched films.

The samples for FT-IR studies were prepared as follows. The polymer blends were cast on $\mathrm{KBr}$ windows using $c a .1$ wt\% solution (the same solvent as in film preparation). Then the samples were placed in a vacuum oven and heated slowly. The final temperature was at $125^{\circ} \mathrm{C}$ and the samples were vacuumed for $18 \mathrm{~h}$ to remove all the residual solvent. Spectra for all the samples were obtained at room temperature with an average of 64 scans at a resolution of $4 \mathrm{~cm}^{-1}$. The wavelength range was from 400 to $4000 \mathrm{~cm}^{-1}$.

\section{RESULTS AND DISCUSSION}

All the polymers after preparation appeared to be transparent without any observable phase separation indicating possible miscibility.

\section{Glass Transition Temperatures}

The second thermal scans from 30 to $180^{\circ} \mathrm{C}$ of the three polymer blends are shown in Figures 1, 2, and 3 in the order of iPMMA, aPMMA, and sPMMA, respectively. According to Figures 1 to 3 , there is a single glass transition temperature for each composition of the blend. Therefore all the three polymer blends are miscible based on the transparency of the films and composition dependent glass transition temperatures. The $T_{\mathrm{gsc}}$ and $T_{\mathrm{gq}}$ values of the three polymer blends are listed in Table I. In most cases, the difference between the $T_{\mathrm{gsc}}$ and $T_{\mathrm{gq}}$ values is within experimental error $\left(\mathrm{ca} .4^{\circ} \mathrm{C}\right)$. Since the $T_{\mathrm{gq}}$ values are considered independent of thermal history so they are used for the following discussion.

All the $T_{\mathrm{gq}}$ values of the three polymer blends plotted vs. phenoxy composition are shown in Figure 4. For the sPMMA/phenoxy blends, $T_{\mathrm{g}}$ was found to obey weight average predictions well. Since the $T_{\mathrm{g}}$ values of aPMMA and phenoxy are very close, no comment can be made in the $T_{\mathrm{g}}$ trend. However, the $T_{\mathrm{g}}$ values of the iPMMA/phenoxy blends are below weight average. For very weak or no specific intermolecular interactions, the Gorden-Taylor ${ }^{15}$ equation adequately predicts the $T_{\mathrm{g}}$ for miscible polymer blends. The Gorden-Taylor equation is shown below as in eq 1

$$
T_{\mathrm{g}}=\left(W_{1} T_{\mathrm{g} 1}+k W_{2} T_{\mathrm{g}}\right) /\left(W_{1}+k W_{2}\right)
$$

where $W_{1}$ and $W_{2}$ denote the weight fractions of the components 1 and $2, T_{\mathrm{g}}, T_{\mathrm{g} 1}$ and $T_{\mathrm{g} 2}$ are the glass transition temperatures of a blend, polymers 1 and 2 , respectively and $k$ is a parameter. A value of $k=0.38$ was found to fit the data points well in the blends of iPMMA and phenoxy.

Paul et $a l^{13}$ also studied PMMA/phenoxy blends, the $T_{\mathrm{g}}$ values of their PMMA and phenoxy are $c a .108^{\circ} \mathrm{C}$ and $89^{\circ} \mathrm{C}$, respectively. $M_{w}$ and $M_{n}$ of their phenoxy are 80000 and 23000 . Our phenoxy's $T_{\mathrm{g}}$ is higher than theirs likely due to different $T_{\mathrm{g}}$ determinations or difference in molecular weight. A single composition dependent but below weight average $T_{\mathrm{g}}$ was found in their studies. Their $T_{\mathrm{g}}$ data can also be likely fitted by the GordonTaylor equation.

Therefore no or little $T_{\mathrm{g}}$ elevation was observed in the PMMA/phenoxy blends. Based on the $T_{\mathrm{g}}$ data, it can be concluded that weak or no inter-hydrogen bonding interaction existing between PMMA and phenoxy. This is quite different from a previous investigation ${ }^{16}$ on similar miscible blends composed of PMMA and poly(styrene-co$p$-hydroxystyrene) (PHS) with $15 \mathrm{~mol} \%$ of hydroxystyrene. A $T_{\mathrm{g}}$ elevation caused by strong hydrogen bond in- 


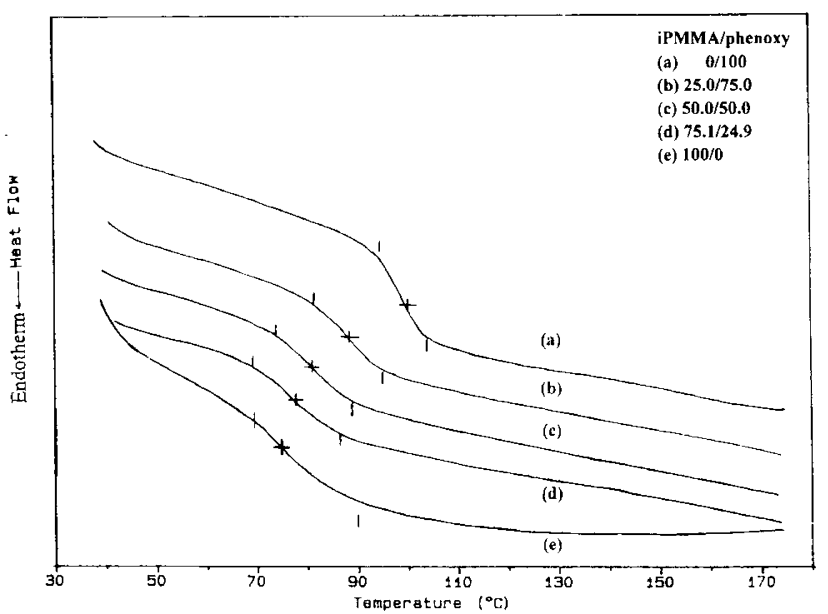

Figure 1. DSC thermograms of iPMMA/phenoxy blends.

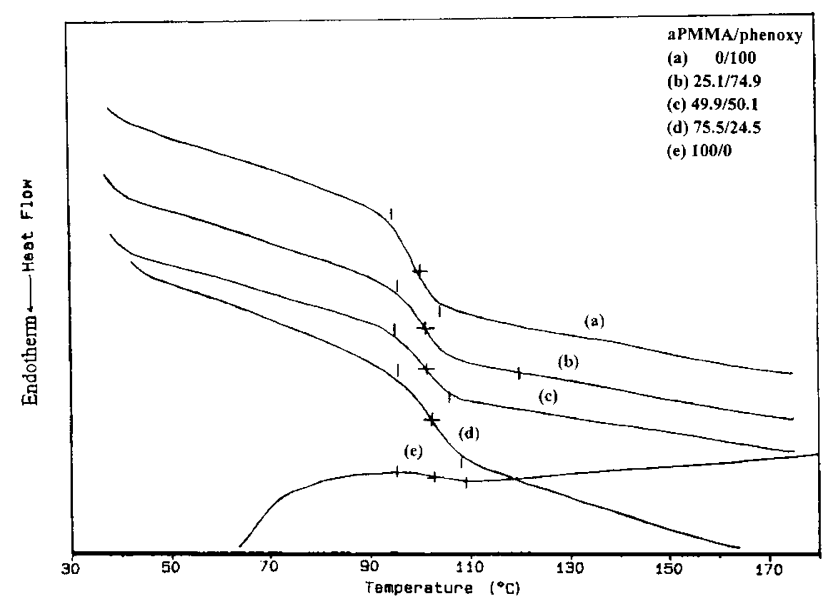

Figure 2. DSC thermograms of aPMMA/phenoxy blends.

teraction between the PMMA carbonyl group and the PHS hydroxyl group was observed.

The glass transition temperature regions $\left(\Delta T_{\mathrm{gq}}\right)$ of the PMMA/phenoxy belnds were calculated as the differences between the onset and the end points of $T_{\mathrm{gq}}$. The calculated results are shown in Figure 5 and also listed in Table I . It is interesting to notice that for the iPMMA/ phenoxy blends no or little broadening of the glass transition temperature regions was observed. Virtually no broadening of the glass transition regions was found in the aPMMA/phenoxy blends because of $T_{\mathrm{g}}$ proximity of the component polymers. However, an obvious broadening $\Delta T_{\mathrm{gq}}$ was detected in the sPMMA/phenoxy blends likely due to composition or density ${ }^{17}$ fluctuations.

\section{Tacticity Effects}

The $m m, m r$, and $r r$ fractions of the iPMMA, aPMMA, and SPMMA were not measured. The simple estimation of $m$ (meso) or $r$ (racemic) fractions from $T_{\mathrm{gq}}$ was reported previously. ${ }^{16}$ The effect of molecular weight of PMMA on $T_{\mathrm{g}}$ is assumed to be negligible. The following two equations describing a linear correlation between $T_{\mathrm{g}}$ and tacticity were used to calculate the meso and racemic fractions of PMMA :

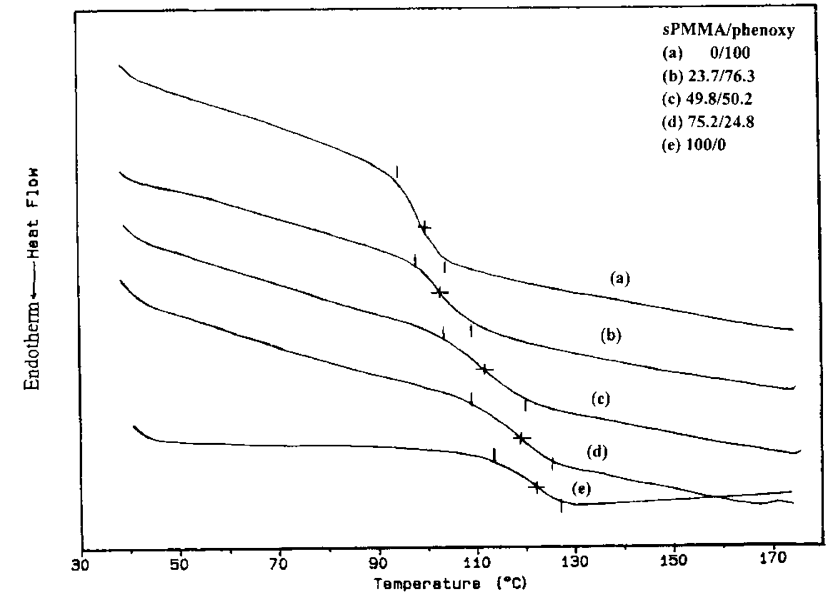

Figure 3. DSC thermograms of SPMMA/phenoxy blends.

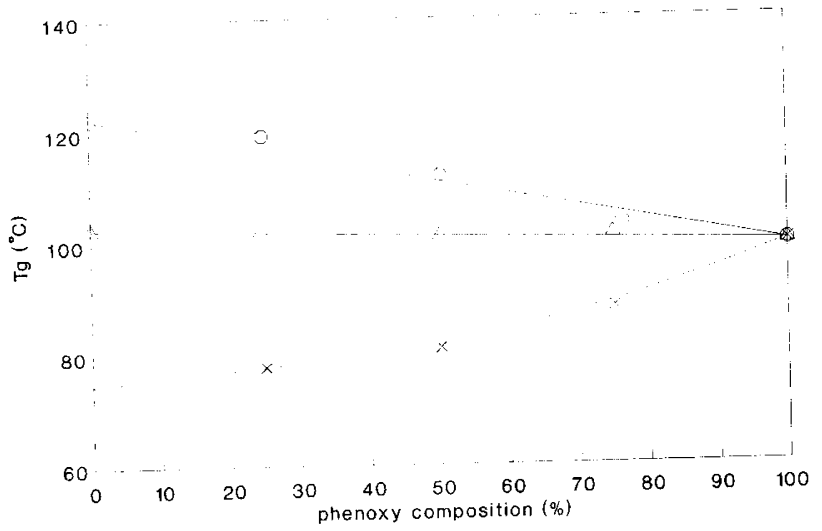

Figure 4. Glass transition temperatures of PMMA/phenoxy blends. $\times$ :iPMMA, $\triangle$ : aPMMA, $\bigcirc$ :sPMMA. - weight average, ---.-according to the Gordon-Taylor eq.

$$
\begin{aligned}
& T_{\mathrm{g}}=41(m)+126(r) \\
& T_{\mathrm{g}}=133.4-76.6(\mathrm{~m})
\end{aligned}
$$

The calculated $m$ and $r$ fractions of iPMMA, aPMMA, and SPMMA are $68.7 \%$ and $31.3 \%, 33.8 \%$ and $66.2 \%$, and $9.3 \%$ and $90.7 \%$, respectively. The error is about $5-8 \%$.

\section{FT-IR Studies on the as-Cast Films}

Our main focus is on hydrogen bonding interaction, therefore only the absorption of the carbonyl and hydroxyl groups is reported in the following paragraph.

The FT-IR spectra of the iPMMA/phenoxy blends in the carbonyl absorption region (from 1650 to $1800 \mathrm{~cm}^{-1}$ ) are shown in Figure 6 for representation because the spectra of the other two blends are about the same. In Figure 6, only the free carbonyl group stretching peak at 1729-1734 was observed (the peak value listed in Table II ). However, Coleman and Moskala observed ${ }^{11}$ an increasing intensity of a shoulder centered at approximately $1720 \mathrm{~cm}^{-1}$ with increasing concentration of phenoxy in the blends with poly(caprolactone). They assigned this band $\left(1720 \mathrm{~cm}^{-1}\right)$ to a hydrogen bonded carbonyl group. They also detected a subtle shift to lower 


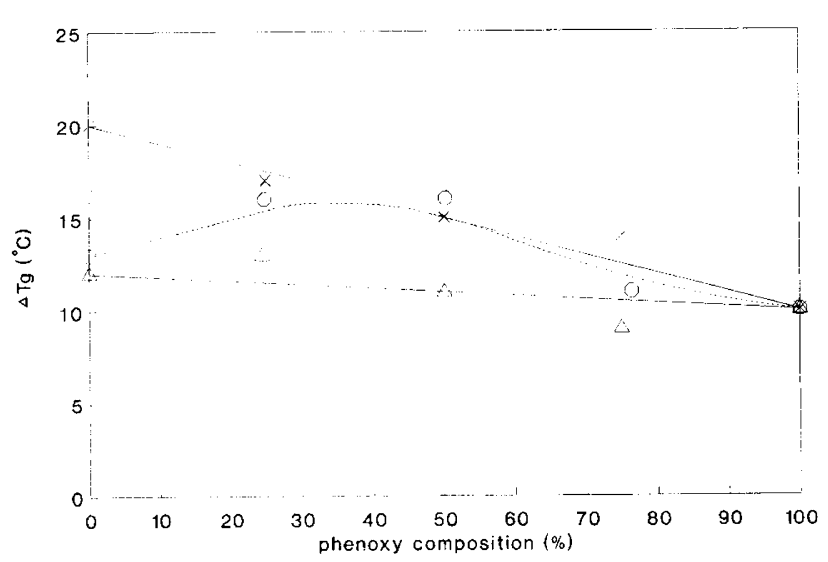

Figure 5. Glass transition temperature regions of PMMA/phenoxy blends. $\times$ :iPMMA, $\triangle$ :aPMMA, $\bigcirc$ :sPMMA. — weight average.

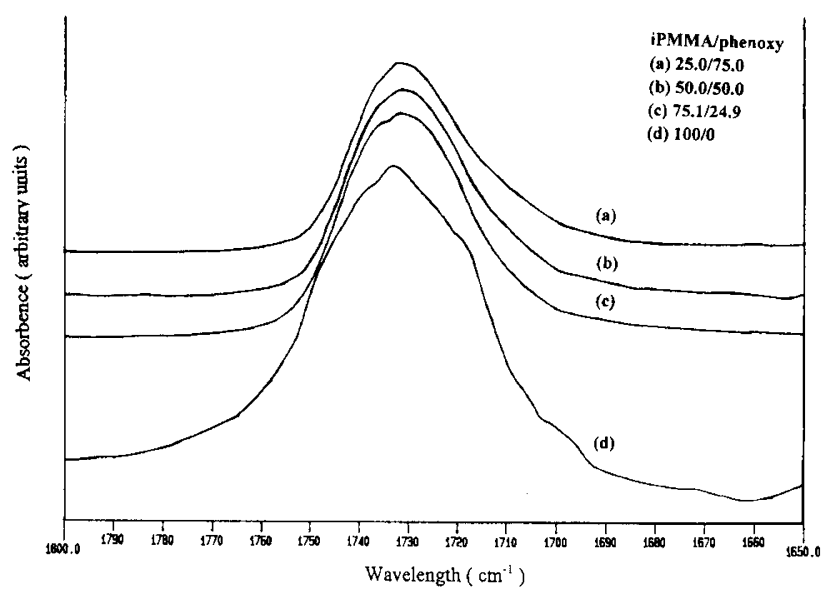

Figure 6. FT-IR spectra of iPMMA/phenoxy blends in the 1650$1800 \mathrm{~cm}^{-1}$ region.

frequency in the major peak at $1735 \mathrm{~cm}^{-1}$ caused by an artifact arising from the contribution of the broad underlying band at $1720 \mathrm{~cm}^{-1}$. From both Figure 6 and Table II and taking into consideration the IR resolution, it is concluded that there is no or little indication of hydrogen bonding from carbonyl absorption.

However, the spectra from the hydroxyl absorption show some indications of $\mathrm{OH}-\mathrm{O}=\mathrm{C}$ interaction. The reason for no indication of hydrogen bonding observed in carbonyl absorption but some indications in hydroxyl absorption may be the following: The peak shift of hydrogen bonded carbonyl group is much smaller ( $c a$. 9-17 $\mathrm{cm}^{-1}$ in ref 11 and 16 and likely smaller in this study) compared to that of hydroxyl group bonded to carbonyl group ( $c a .90 \mathrm{~cm}^{-1}$ in ref 16). It is quite possible that the shoulder caused by hydrogen bonding in carbonyl group is masked by the free carbonyl absorption. The hydroxyl absorption spectra (from 3200 to $3700 \mathrm{~cm}^{-1}$ ) of the studied blends are shown in Figures 7, 8, and 9. For phenoxy (plotted in all the three Figures), there is one broad peak centered at $3418 \mathrm{~cm}^{-1}$ indicative of extensively hydrogen bonded hydroxyl groups. This is entirely consistent with self-association of the polymer chains through intermolecular hydrogen bonding of the hydroxyl groups.
Table II. Peak positions of carbonyl and hydroxyl stretching regions in PMMA/phenoxy blends

\begin{tabular}{ccc}
\hline & $\mathrm{C}=\mathrm{O}$ & $\mathrm{OH}$ \\
\hline (1) iPMMA/Phenoxy & & \\
$100 / 0$ & 1734 & $\ldots$ \\
$75.1 / 24.9$ & 1732 & 3516 \\
$50.0 / 50.0$ & 1732 & 3507 \\
$25.0 / 75.0$ & 1732 & 3452 \\
$0 / 100$ & $-\cdots$ & 3418 \\
(2) aPMMA/Phenoxy & & \\
100/0 & 1734 & $\ldots$ \\
$75.5 / 24.5$ & 1729 & 3507 \\
$49.9 / 50.1$ & 1732 & 3490 \\
$25.1 / 74.9$ & 1732 & 3424 \\
$0 / 100$ & $-\cdots$ & 3418 \\
100/0 & & \\
$75.2 / 24.8$ & 1734 &.-- \\
$49.8 / 50.2$ & 1730 & 3527 \\
$23.7 / 76.3$ & 1731 & 3509 \\
$0 / 100$ & 1731 & 3500 \\
& $-\cdots$ & 3418 \\
\hline
\end{tabular}

There should likely be some unassociated (free) hydroxyl groups present in the phenoxy shown as a shoulder at $3570-3650 \mathrm{~cm}^{-1}$ but not detected here. The reason may be due to too little absorption. The spectrum of phenoxy was previously thought to be composed of the free hydroxyl group absorption appearing in the aforementioned region and the hydrogen bonded hydroxyl group absorption at $3300-3550 \mathrm{~cm}^{-1} \cdot{ }^{18}$ It was later confirmed that there are three Gaussian peaks in the hydroxyl stretching region, instead of just two major spectral components observed visually. Therefore hydrogen bonded hydroxyl band could be further resolved into a 'dimer' hydrogen bonded hydroxyl peak at $3430-3550 \mathrm{~cm}^{-1}$ and the 'multimer' hydrogen bonded hydroxyl peak around $3250-3380 \mathrm{~cm}^{-1} .19$

When PMMA is added to phenoxy, the carbonyl group of PMMA also will form hydrogen bond with the hydroxyl group of phenoxy. Therefore in the blends of PMMA and phenoxy, hydrogen bonds have two types of interactions (i.e., $\mathrm{OH}-\mathrm{OH}$ and $\mathrm{OH}-\mathrm{O}=\mathrm{C}$ interactions.) Actually, $\mathrm{OH}-\mathrm{O}=\mathrm{C}$ interactions play a major role in making the blends miscible. Interestingly upon mixing with PMMA, the broad hydrogen bonded hydroxyl band of the phenoxy (the major peak) was observed to be shifted to higher frequencies as a function of increasing PMMA concentration. In addition to Figures 7 to 9, this can be seen more directly from the hydroxyl peak position listed in Table II. The meaning of a higher frequency shift is expounded in the following paragraph. There is also a minor peak at a lower frequency both observed in Figures 7 and 9 (iPMMA and sPMMA blends) probably due to more $\mathrm{OH}-\mathrm{O}=\mathrm{C}$ interaction or residual $\mathrm{OH}-\mathrm{OH}$ interaction. However, no observable minor peak was found in Figure 8 (aPMMA blends). In the Figure 6 of Coleman and Moskala's study ${ }^{11}$, we also found a minor peak at lower frequencies in their blends of poly(caprolactone) and phenoxy. Although they did not give any explanation concerning this peak, we believe the underlying reason is similar to ours.

The shift of an essentially uncoupled vibrational mode of a polymer to higher frequency upon mixing with another miscible polymer is not without precedent. In one instance, Coleman et al. ${ }^{11}$ reported the broad hydrogen 


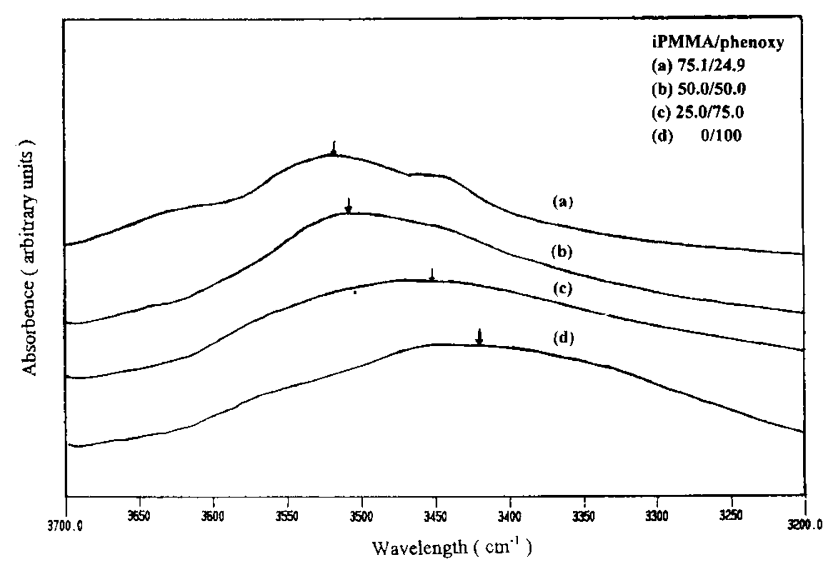

Figure 7. FT-IR spectra of iPMMA/phenoxy blends in the 3200$3700 \mathrm{~cm}^{-1}$ region.

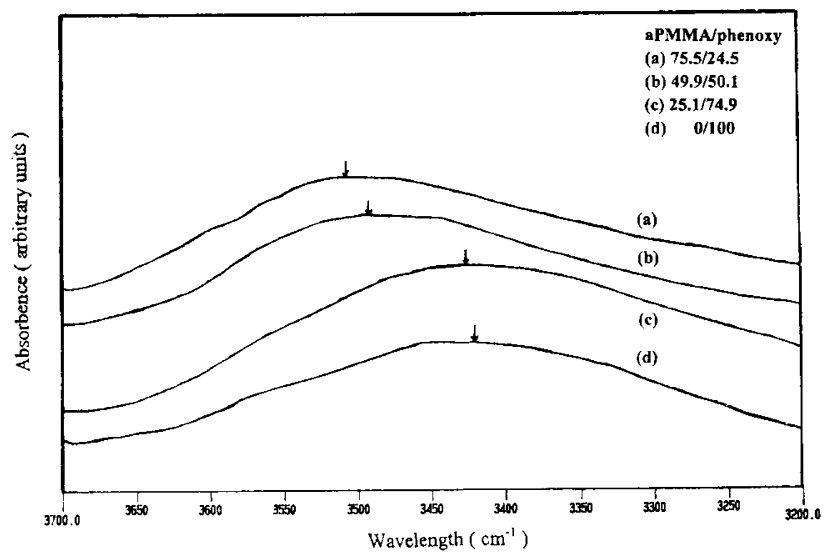

Figure 8. FT-IR spectra of aPMMA/phenoxy blends in the 3200 $-3700 \mathrm{~cm}^{-1}$ region.

bonded band of the phenoxy is observed to shift to higher frequencies as a function of increasing poly(caprolactone) (PCL) concentration in the blends of phenoxy and PCL. Because our system is analogous to their studies, therefore we adopted their interpretations. In the PMMA/phenoxy blends, a significant fraction of the phenoxy hydroxyl groups are presumably associated with the PMMA carbonyl groups. However, in this case the overall relative strength of the hydrogen bonds is less than that of the self-associated phenoxy hydroxyl groups. Nevertheless, there is still a shift to lower frequency relative to that of the free hydroxyl.

A question that one may ask is, "if the hydrogen bonding interaction is weaker in the blends compared with that of the self-associated pure phenoxy, why do these two polymers form a miscible amorphous blend?" In simple terms, for a polymer-polymer mixture of a certain composition to be miscible, the overall free energy of mixing must be equal to or less than zero. The changes in entropy upon mixing amorphous macromolecular species is believed to be relatively small but it is generally positive and contributes a negative factor to the free energy of mixing. In the PMMA/phenoxy blends one would have to consider the enthalpic and entropic changes occurring upon forming a miscible mixture compared to both original pure amorphous polymers. Although the infrared spectroscopic results presented above indicate

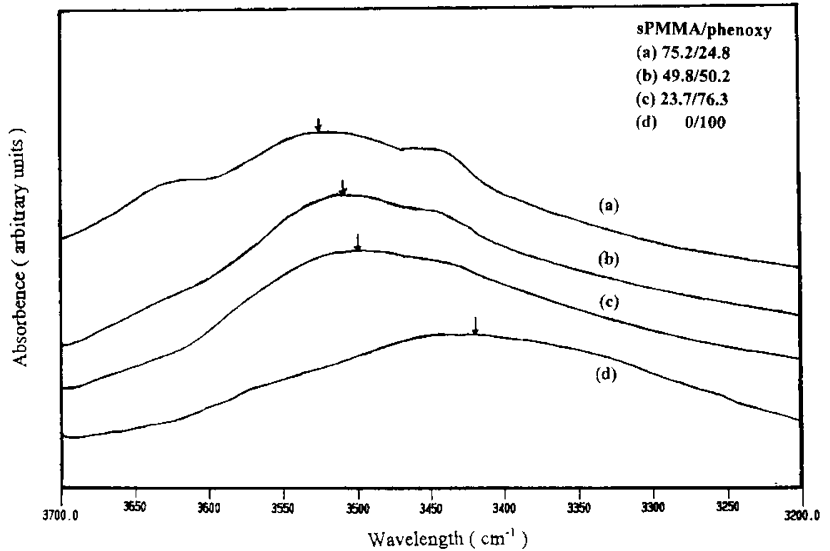

Figure 9. FT-IR spectra of sPMMA/phenoxy blends in the 3200 $-3700 \mathrm{~cm}^{-1}$ region.

that the strength of the hydrogen bonding interaction in pure phenoxy is stronger than that occurring in the blends, it is the balance between the changes in enthalpy and entropy is important. Given that the PMMA/ phenoxy blends are miscible, this must imply that the resulting balance between the enthalpy and entropy of mixing leads to an overall decrease in the free energy of the system.

According to the magnitude of the peak shift in Table II , the degree of hydrogen bonding between PMMA and phenoxy should follow the order: sPMMA >iPMMA $>$ aPMMA. Using the PMMA/phenoxy (ca. 50/50) for example, the peak shift of SPMMA, iPMMA and aPMMA are calculated to be 91,89 , and $72 \mathrm{~cm}^{-1}$, respectively. It is interesting to compare the results of Figures $7-9$ and Table II to those of the PMMA/PHS blends. ${ }^{16}$ Figure 10 (taken from Figure 10 of ref 16) shows the hydroxyl absorption of the iPMMA/PHS blends for representation. The free hydroxyl group absorption of PHS is at 3535 $\mathrm{cm}^{-1}$, and the self-associated hydroxyl group absorption at $3445 \mathrm{~cm}^{-1}$. The peak shift of $\mathrm{OH}-\mathrm{OH}$ interaction in phenoxy is $c a .192-232 \mathrm{~cm}^{-1}$ and that in PHS is much smaller, $90 \mathrm{~cm}^{-1}$. Based on the magnitude of peak shift, the $\mathrm{OH}-\mathrm{OH}$ interaction of phenoxy is stronger than that of PHS. Therefore phenoxy has a lower degree of free hydroxyl groups than PHS. Because the strength of the $\mathrm{OH}$ $-\mathrm{O}=\mathrm{C}$ interaction between PHS and PMMA is comparable to $\mathrm{OH}-\mathrm{OH}$ interaction of PHS, the hydroxyl band hydrogen bonded either to hydroxyl or carbonyl group has almost the same wavelength. Also according to ref 16 , iPMMA and aPMMA have more effective hydrogen bonding with PHS than SPMMA based on the IR evidence. Therefore the order of the inter-hydrogen bonding between PMMA and PHS is different from that between PMMA and phenoxy. Comparing the current results with ref 16 , it is concluded that the inter-hydrogen bonding in the PMMA/phenoxy blends is not as strong as that in the PMMA/PHS blends. Based on the abovementioned results, the inter-hydrogen bonding $(\mathrm{OH}-\mathrm{O}=$ C) and intra-hydrogen bonding $(\mathrm{OH}-\mathrm{OH})$ interactions of the PMMA/phenoxy and PMMA/PHS blends have the following order : $\mathrm{OH}-\mathrm{OH}$ (phenoxy) $>\mathrm{OH}-\mathrm{OH}(\mathrm{PHS}) \sim \mathrm{O}$ $\mathrm{H}-\mathrm{O}=\mathrm{C}(\mathrm{PMMA} / \mathrm{PHS})>\mathrm{OH}-\mathrm{O}=\mathrm{C}(\mathrm{PMMA} /$ phenoxy $)$.

The reason for the order of the inter-hydrogen bonding between each tactic PMMA and phenoxy may be similar 


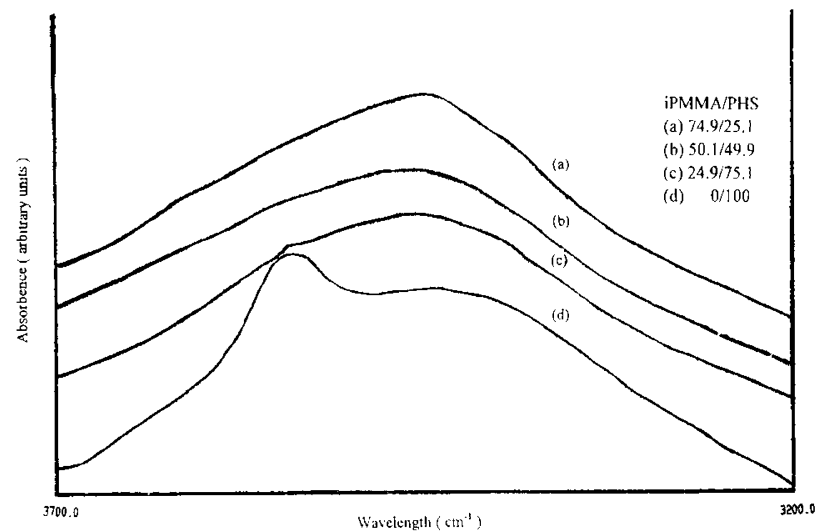

Figure 10. FT-IR spectra of iPMMA/PHS blends in the 3200$3700 \mathrm{~cm}^{-1}$ region(taken from Figure 10 of ref 16 ).

to previous investigation. ${ }^{16}$ iPMMA, aPMMA, and sPMMA all have $m m$ (isotactic), $m r$ (heterotactic), and $r r$ (syndotactic) fractions but in very different degrees. Taking into account that part of $m$ and $r$ fractions belong to $m r$ fractions, the following comments can be drawn. It is obvious that sPMMA is composed mainly of $r r$ fractions and has very few $m m$ and $m r$ fractions. iPMMA consists of about more than half of $\mathrm{mm}$ fractions and $\mathrm{mr}$ and $\mathrm{rr}$ fractions make up the rest. It is likely that aPMMA has more than $50 \%$ of $r r$ fractions and the rest is $\mathrm{mm}$ and $\mathrm{mr}$ fractions. From the experimental results of this study, it is inferred that phenoxy forms more hydrogen bonds with $m m$ or $r r$ fractions of PMMA than with $m r$ fractions. It is also likely that $\mathrm{mm}$ fractions of PMMA form more hydrogen bonds with phenoxy than $r r$ fractions of PMMA since iPMMA has a higher degree of hydrogen bonding with phenoxy than aPMMA.
Acknowledgment. The support by the National Science Council of Taiwan, R.O.C., Grant NSC-88-2216-E041-001 is greatly appreciated.

\section{REFERENCES}

1. E. Roerdink and G. Challa, Polymer, 19, 173 (1978).

2. E. Roerdink and G. Challa, Polymer, 21, 509 (1980).

3. A. P. A. M. Eijkelenboom, W. E. J. R. Mass, W. S. Veeman, G. H. Werumeus Buning, and J. M. J. Vankan, Macromolecules, 25, 4511 (1992).

4. G. Ramana Rao, C. Castiglioni, M. Gussoni, G. Zeroi, and E. Martuscelli, Polymer, 26, 811 (1985).

5. E. John and T. Ree, J. Polym. Sci. Polym Chem. Ed., 28, 385 (1990).

6. C. Silvestre, S. Cimmino, E. Martuscelli, F. E. Karasz, and W. J. MacKnight, Polymer, 28, 1190 (1987).

7. J. W. Schurer, A. de Boer, and G. Challa, Polymer, 16, 201 (1975).

8. E. J. Vorenkamp, G. ten Brinke, J. G. Meijer, H. Jager, and G. Challa, Polymer, 26, 1725 (1985).

9. M. Suess, K. Kressler, and H. W. Krammer, Polym. Bull., 16 , 371 (1986).

10. J. M. Martinez, J. I. Equiazabal, and J. Nazabal, J. Macromol. Sci. Phys., B30, 345 (1991).

11. M. M. Coleman and E. J. Moskala, Polymer, 24, 251 (1983).

12. M. Iriarte, E. Epsi, A. Etxeberria, M. Valero, M. J. Fernandez -Berridi, and J. J. Iruin, Macromolecules, 24, 5545 (1991).

13. J. S. Chiou and D. R. Paul, J. Appl. Polym. Sci., 42, 279 (1991).

14. B. K. Kim and C. H. Choi, Polymer, 37, 807 (1996).

15. M. Gordon and J. S. Taylor, J. Appl. Chem., 2, 493 (1952).

16. W. P. Hsu and C. F. Yeh, J. Appl. Polym. Sci., 73, 431 (1999) and references therein.

17. R. E. Prud'homme, Polym. Eng. Sci., 22, 90 (1982).

18. D. L. Paria, G. M. Lampman, and G. S. Kriz, "Introduction to Spectroscopy : A Guide for Students of Organic Chemistry", Saunders College Publishing, Philadelphia, PA, 1979.

19. M. M. Coleman, X. Yang, and P. C. Painter, Macromolecules, 25, 4414 (1992). 